\title{
Cephalosporin Resistance in Neisseria gonorrhoeae Infections
}

\author{
Robert D. Kirkcaldy, MD, MPH, \\ Division of STD Prevention, Centers for Disease Control and Prevention, Atlanta, Georgia; \\ Gail A. Bolan, MD, \\ Division of STD Prevention, Centers for Disease Control and Prevention, Atlanta, Georgia; \\ Judith N. Wasserheit, MD, MPH \\ Department of Global Health, University of Washington, Seattle.
}

In Reply: We agree with Drs Klausner and Kerndt that antibiotic stewardship is an important component of controlling antibiotic resistance, and we support such efforts as sound clinical and public health practice. For many bacterial pathogens, antibiotic consumption appears to promote the emergence of resistance, and judicious antibiotic use may reduce the prevalence of resistance. ${ }^{1}$

However, for several reasons, it is not at all clear that improved antibiotic stewardship in the United States would prevent the emergence of cephalosporin resistance in $N$ gonorrhoeae. First, gonococcal resistance is a global phenomenon, and importation of resistant strains from other countries appears to play a large role in the emergence of resistance in the United States. Gonococcal resistance phenotypes tend to emerge initially in East Asia before spreading globally. ${ }^{2}$ When resistance has emerged in the United States, it has appeared first in geographic regions in relative proximity to Asia such as Hawaii and the West Coast.

Second, resistance has emerged first in the western United States, yet the West has the lowest per capita antimicrobial prescription sales of all regions. ${ }^{3}$ In addition, unlike some other bacterial pathogens, $N$ gonorrhoeae maintains genetic resistance determinants even after the apparent removal of antibiotic selection pressure; some resistance determinants may actually provide a fitness advantage, even in the absence of antibiotics. ${ }^{4,5}$

The decline or discontinuation in the use of penicillin or fluoroquinolones for treatment of gonorrhea has not appreciably changed the prevalence of $N$ gonorrhoeae resistance to these antibiotics in the United States. ${ }^{6}$ It is possible that for selected antibiotics, such as macrolides, local antibiotic usage might influence resistance patterns and that domestic selection pressure due to antibiotic use for other indications might contribute to gonococcal resistance to some degree. This has not been clearly demonstrated. More research on the relationship between domestic antibiotic prescribing and the emergence or persistence of gonococcal resistance may prove helpful.

rkirkcaldy@cdc.gov.

Conflict of Interest Disclosures: The authors have completed and submitted the ICMJE Form for Disclosure of Potential Conflicts of Interest and none were reported. 
We agree with Klausner and Kerndt that real-time antimicrobial susceptibility testing or the addition of molecular markers of genetic resistance determinants to existing nucleic acid amplification tests could have the potential to guide the choice of antibiotics, so that ideally the most effective antibiotic can be prescribed at the time of diagnosis. At this time, however, antimicrobial susceptibility testing for $N$ gonorrhoeae is not widely available. It requires culture of the live organism, and culture is done infrequently in most clinical settings and laboratories.

Incorporating assays for well-characterized resistance genotypes, such as for ciprofloxacin resistance, into nucleic acid amplification tests does hold promise. Research to develop both nonculture tests for gonococcal antimicrobial susceptibility and new antimicrobial agents will be a key component of an effective response to the threat of antimicrobial-resistant $N$ gonorrhoeae.

\section{References}

1. Willmann M, Marschal M, Hölzl F, et al. Time series analysis as a tool to predict the impact of antimicrobial restriction in antibiotic stewardship programs using the example of multidrug-resistant Pseudomonas aeruginosa. Antimicrob Agents Chemother. 2013;57(4):1797-1803. [PubMed: 23380719]

2. Ross JDC. Fluoroquinolone resistance in gonorrhoea: how, where and so what. Int J STD AIDS. 1998;9(6):318-322. [PubMed: 9671243]

3. Hicks LA, Suda KJ, Robert RM, Hunkler R, Taylor TH, Danziger LH. Antimicrobial prescription data reveal wide geographic variability in antimicrobial use in the United States, 2009. Presented at: 48th Annual Meeting of the Infectious Disease Society of America; October 21-24, 2010; Vancouver, British Columbia, Canada Abstract 339.

4. Unemo M, Shafer WM. Antibiotic resistance in Neisseria gonorrhoeae: origin, evolution, and lessons learned for the future. Ann N Y Acad Sci. 2011;1230: E19-E28. [PubMed: 22239555]

5. Kunz AN, Begum AA, Wu H, et al. Impact of fluoroquinolone resistance mutations on gonococcal fitness and in vivo selection for compensatory mutations. J Infect Dis. 2012;205(12):1821-1829. [PubMed: 22492860]

6. Centers for Disease Control and Prevention. Sexually transmitted disease surveillance 2011 http:// www.cdc.gov/std/stats11/default.htm. Accessed February 15, 2013. 